\title{
表面活性剂用于废水处理研究的新进展
}

\author{
赵微微 $a, b$ 王毅琳*, $, a, b$ \\ ( ${ }^{a}$ 中国科学院化学研究所 胶体界面与化学热力学实验室 北京 100190) \\ ${ }^{b}$ 中国科学院大学 北京 100049)
}

\begin{abstract}
摘要 随着工业化和农业现代化的迅速发展, 水污染问题逐渐成为人们关注的焦点. 大量工业废水的随意排放造成水 体污染, 导致可利用的淡水资源十分匮乏. 因此, 如何低成本, 安全、高效地处理工业废水, 提高水资源的重复利用效 率已经成为亟待解决的重要问题. 近年来, 基于表面活性剂的分离技术在工业和分析领域取得了突破性进展, 其绿色 环保和低能耗的特点为废水处理提供了理想的选择. 介绍了基于表面活性剂的废水处理技术一一胶束增强超滤、表面 活性剂改性的固相吸附和基于表面活性剂液液相分离的提取技术的原理以及研究进展，旨在为研究人员提供参考，进 一步推动污水处理技术的发展.
\end{abstract}

关键词＼cjkstart表面活性剂; 废水处理; 液液相分离; 胶束增强超滤; 吸附

\section{Development of Surfactant Application in Wastewater Treatment}

$$
\text { Zhao, Weiwei }^{a, b} \quad \text { Wang, Yilin }{ }^{*, a, b}
$$

( ${ }^{a}$ Key Laboratory of Colloid, Interface and Chemical Thermodynamics, Institute of Chemistry, Chinese Academy of Sciences, Beijing 100190)

$\left({ }^{b}\right.$ University of Chinese Academy of Sciences, Beijing 100049)

\begin{abstract}
Water is the most important and essential component for the existing activities of human beings, animals and plants. It is estimated that the total amount of water on the earth is about 1.3 billion tons, but $97 \%$ of that is salty ocean water and not suitable for drinking. With the rapid growth of population, industrialization and agricultural modernization and other geological and environmental changes, the water environment is deteriorating continuously. Water pollution and water shortage are two of the most important environmental problems in the world. Consequently, water pollution has become a critical issue in recent years. Pollutants in wastewater include organic, inorganic, biological compounds. As many of them have serious toxicity and even show carcinogenic, the release of considerable amount of wastewater into environment causes damages to human being and aquatic conditions, and further leads to the shortage of water resources. Therefore, the need for wastewater treatment in a low-cost, safe and efficient way and improving the reuse efficiency of water resources have become a must. In recent years, surfactant-based separation techniques have made a great progress in industrial and analytical areas. It offers many advantages including low-energy consumption and environment protection, and has been proved efficient in the separation of many inorganic and organic pollutants. To enhance the application of surfactant-based separation techniques in wastewater treatment, it is very important to have a better understanding of the mechanisms involved in this process. The mechanism and development of surfactant-based wastewater treatment techniques, including micelle-enhanced ultrafiltration (MEUF), surfactant-modified solid phase adsorption and surfactant-based liquid-liquid phase separation are summarized. The effects of the surfactant characteristics, the chemistry of the pollutants and the solution conditions used in experiments on the extract kinetics and efficiencies are discussed. This review aims to provide reference and inspiration for researchers and promote the development of wastewater treatment technologies.

Keywords surfactant; wastewater treatment; coacervation; micelle-enhanced ultrafiltration; adsorption
\end{abstract}

\section{1 引言}

水是人类赖以生存的重要资源之一. 随着人口的增 长和工业化的快速发展, 水资源质量不断下降, 严重地 影响了社会的发展, 水污染问题逐渐成为全球范围关注 的焦点 ${ }^{[13]}$. 根据污染物来源的不同, 污水中污染物主 要包括重金属离子、有机污染物、生物制剂和辐射物质
等. 这些污染物质不仅会对水体环境造成破坏，影响水 体生态系统，导致水质恶化，难降解的有机污染物还会 经过代谢在人体中富集, 对人类健康造成威胁. 例如, 砷 $(\mathrm{As})$ 、镉 $(\mathrm{Cd})$ 、钴 $(\mathrm{Co})$ 、铬 $(\mathrm{Cr}) 、$ 铜 $(\mathrm{Cu}) 、$ 铅 $(\mathrm{Pb})$ 、镍 $(\mathrm{Ni})$ 和钒 $(\mathrm{V})$ 等重金属离子会损害人体的神经系统、造血 系统以及肾脏等多种器官, 具有较强的致癌性 ${ }^{[4]}$; 多种 染料分子和多环芳烃 PAH(如葱、䒢等有机物 $)^{[5]}$ 已被证

\footnotetext{
* E-mail: yilinwang@iccas.ac.cn; Tel.: 010-82615802; Fax: 010-82615802

Received May 17, 2019; published July 10, 2019.

Project supported by the National Natural Science Foundation of China (No. 21633002).

项目受国家自然科学基金(No. 21633002)资助.
} 
实对动物和人体有致癌作用. 因此, 如何实现低成本、 高效率地处理工业废水, 提高水资源的重复利用效率已 经成为亟待解决的重要问题.

目前对于废水的处理方法大致分为四类: 物理化学 方法、生物学方法、核处理方法以及声学、电学、电磁 学方法 ${ }^{[6 ~ 19]}$. 具体方法包括过滤、超滤、透析、反向渗 透、离子交换、溶剂萃取法、氧化法、吸附法、絮凝法、 泡沫浮选法、光化学反应、活性污泥、有氧和庆氧处理 法、细菌处理法、核辐射法、电渗析法、超声处理法和 磁分离法等. 许多方法在对废水分析和处理中发挥着至 关重要的作用, 但是处理成本、操作方法、处理效率等 因素在一定程度限制了部分方法的应用. 因此, 寻找新 型安全环保的废水处理方法, 降低有机溶剂的消耗, 实 现对污染物的分离和回收, 成为近年来研究的重要课 题, 具有广泛的应用前景.

表面活性剂分子是一类具有亲水基团和疏水基团 的有机化合物, 其独特的结构使其活跃于表/界面, 能够 显著降低表/界面张力; 在溶液中, 表面活性剂高于一定 浓度时能够形成有序的组装体 ${ }^{[20,21]}$. 因此, 表面活性剂 具有润湿、乳化、起泡、增溶、防腐、分散、洗涤和抗 静电等一系列物理化学性能, 已被广泛地应用在日化、 石油、食品、农业、卫生、环境、新型材料等领域, 具

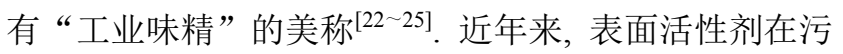
水处理中的应用受到人们广泛的关注, 基于表面活性剂 的分离技术在工业领域和分析领域取得了很多新进展. 基于表面活性剂的分离方法具有低能耗、绿色环保等特 点, 其中胶束增强超滤(micelle-enhanced ultrafiltration, MEUF)、表面活性剂改性固定相提取以及液液相分离提 取方法具有代表性. 本综述介绍了基于表面活性剂的废 水处理技术一一胶束增强超滤、表面活性剂改性的固相 吸附和基于表面活性剂液液相分离的提取技术的原理 以及研究进展, 旨在为研究人员提供参考, 进一步推动 污水处理技术的发展.

\section{2 胶束增强超滤}

\section{1 胶束增强超滤原理}

表面活性剂分子由亲水基团和疏水基团两部分组 成, 根据亲水基团性质的不同, 可以分为非离子表面活 性剂、阴离子表面活性剂、阳离子表面活性剂和两性离 子表面活性剂. 当表面活性剂在水溶液中浓度大于某一 临界值时, 表面活性剂分子可以自组装形成胶束, 此临 界值被称为临界胶束浓度(Critical Micelle Concentration, $\mathrm{CMC})^{[26]}$. 改变表面活性剂的分子结构, 能够形成囊泡、 蠕虫状胶束、球形胶束等多种聚集体 ${ }^{[27,28]}$. 以离子表面 活性剂为例, 表面活性剂形成的胶束由疏水性内核和亲 水性外层组成: 内部由表面活性剂分子的疏水尾链相互 交联形成疏水性微区，外层主要包括水合的亲水头基以 及结合水. 胶束的疏水内核与液态烃相似, 但粘度更高,
对于疏水性物质具有较好的增溶能力. 根据亲-疏水性 不同，胶束对于不同物质的增溶发生在四个部位：胶 束-水界面、亲水头基之间、胶束的栅栏层和胶束的疏 水内核. 离子型污染物通过静电相互作用与带相反电荷 的表面活性剂头基结合，增溶发生在胶束的表面; 而有 机物根据结构的不同，可以被增溶在胶束的栅栏层或疏 水内核.

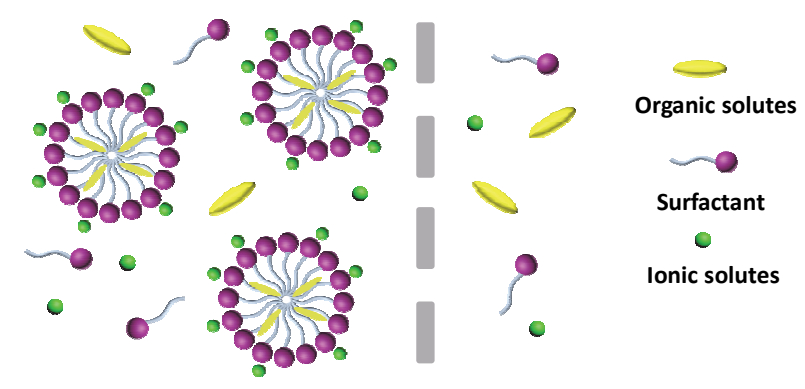

图 1 胶束增强超滤提取离子型污染物和有机物的原理

Figure 1 Mechanism of removal of ionic solutes and organic solutes by MEUF

基于胶束的增溶特性, 胶束增强超滤(MEUF)技术 将表面活性剂和滤膜结合，用于分离废水中低浓度的有 机物(如磷酸二丁酯、磷酸三丁酯、三卤甲烷等)和金属 离子(如镉、铅、锌等), 是一项非常有前景的废水处理 技术. 其原理是向废水中加入一定量的表面活性剂, 形 成的胶束增溶了被分离物, 当废水通过超滤膜时, 未被 增溶的金属离子和有机物以及表面活性剂单体可以透 过滤膜, 进入下一步循环, 而增溶了金属离子和有机物 的胶束由于粒径大于滤膜孔径而被截留, 形成表面活性 剂和污染物的高浓度溶液, 实现了废水净化分离的目的 (图 1). 胶束增强超滤技术的滤膜孔径在 $1.0 \sim 10.0 \mathrm{~nm}$, 所需压力在 97 587 kPa 范围内, 该技术结合了反渗透 膜的高选择性和超滤的高通量的特点, 具有能耗低、驱 动力低的优势. 利用传统的超滤技术处理废水时, 由于 重金属离子的半径为纳米级别, 传统超滤膜孔径在 $2.0 \sim 100.0 \mathrm{~nm}$ 之间, 很难截留金属离子; 对于有机物, 单一的超滤技术只能截留分子大于滤膜孔径的有机污 染物, 因此, 超滤技术通常与光催化 ${ }^{[29,30] 、 \text { 吸附 }}{ }^{[31,32]}$ 等技 术相结合, 提高对于小分子量污染物的截留率. 与传统 的超滤技术相比, 胶束增强超滤技术能够去除废水中较 低浓度的金属离子和有机污染物，操作简单，能耗低， 易实现工业化，具有良好的应用前景和环境效益.

\section{2 表面活性剂的选择}

表面活性剂的选择是影响污染物分离效果的重要 因素。在胶束增强超滤中, 表面活性剂分子应具有 $\mathrm{CMC}$ 值较低、胶束体积较大、在超滤膜表面吸附量小、 对被分离物质增溶能力强以及可生物降解等特点. 首 先，表面活性剂的选取需保证被分离物质/表面活性剂 分子之间具有较强的相互作用. 例如, 去除金属离子时, 
应选取带有相反电荷的阴离子表面活性剂，二者可以通 过静电相互作用有效地结合; 去除金属阴离子以及带负 电荷的有机物(如染料分子)时, 应选择阳离子表面活性 剂; 去除有机物时, 阴离子、阳离子以及非离子表面活 性剂的理论上都适用, 需根据水质进行选择. 其次, 表 面活性剂分子应具有较低的 CMC 值, 可以降低表面活 性剂的用量以及滤液中残留的表面活性剂的浓度. 此 外, 选取可降解的表面活性剂分子, 后处理可以采用生 物降解处理方法, 有效地减少表面活性剂对于环境的污 染. 目前文献中报道的常用的表面活性剂包括聚环氧乙 烷一聚环氧丙烷一聚环氧乙烷三嵌段共聚物(PEO-PPO-

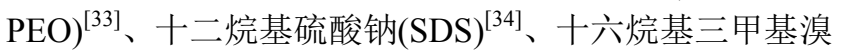
化铵 $(\mathrm{CTAB})^{[35]}$ 、氯化十六烷基吡啶 $(\mathrm{CPC})^{[36]}$ 、脂肪酸甲 酯乙氧基化物(OMC-10 $)^{[37]}$ 及烷基多糖苷(APG) $)^{[38]}$ 等.

\section{3 胶束增强超滤用于去除水中污染物}

胶束增强超滤技术能够去除单一的金属离子. 利用 阴离子型表面活性剂如十二烷基硫酸钠 SDS、十二烷基 醚硫酸钠 SLES 等, 胶束表面带有负电荷, 金属离子可 以通过静电相互作用与胶束结合, 当携带金属离子的胶 束通过超滤膜时会被截留下来, 从而达到去除金属离子 的目的. 例如, 用 $\mathrm{SDS}$ 分别去除 $\mathrm{Cu}^{2+}, \mathrm{Ni}^{2+}, \mathrm{Cd}^{2+}, \mathrm{Zn}^{2+}$ 和 $\mathrm{Pb}^{2+}$ 时, 截留率均可达到 $90 \%$ 以上 ${ }^{[39 \sim 43]}$. 此外, 胶束 增强超滤技术也能同时去除多种金属离子, 如 $\mathrm{Ni}^{2+}$ 和 $\mathrm{Zn}^{2+[44]}, \mathrm{Cd}^{2+} 、 \mathrm{Cu}^{2+}$ 和 $\mathrm{Zn}^{2+[45]}, \mathrm{Pd}^{2+} 、 \mathrm{Cd}^{2+}$ 和 $\mathrm{Zn}^{2+[46]}$, $\mathrm{Cd}^{2+} 、 \mathrm{Cu}^{2+} 、 \mathrm{Co}^{2+}$ 和 $\mathrm{Zn}^{2+[47]}, \mathrm{Fe}^{2+} 、 \mathrm{Cu}^{2+} 、 \mathrm{Cd}^{2+} 、 \mathrm{Zn}^{2+}$ 、 $\mathrm{Ni}^{2+}$ 和 $\mathrm{Mg}^{2+[48]}$ 等. Yang 等 ${ }^{[49]}$ 用 SDS 去除同时含有 $\mathrm{Cr}^{3+}$, $\mathrm{Co}^{2+}, \mathrm{Ni}^{2+}$ 和 $\mathrm{Mg}^{2+}$ 的废水溶液, 各种金属离子的初始浓 度是 $1.0 \mathrm{mmol} \cdot \mathrm{L}^{-1}$, 当表面活性剂和金属离子浓度的比 例 $\mathrm{S} / \mathrm{M}$ 在 5 8 之间时, 金属离子的截留率可以达到 $90 \% \sim 99 \%$, 截留率大小依次是 $\mathrm{Cr}^{3+}>\mathrm{Co}^{2+}>\mathrm{Ni}^{2+}>$ $\mathrm{Mg}^{2+}$. 在去除单一金属离子时, 胶束通过静电作用吸附 金属离子直到饱和, 当废液中存在多种金属离子时, 则 存在竞争作用. 添加螯合剂分子或使用表面活性剂的混 合溶液有助于混合金属离子的去除. Akita 等 ${ }^{[50]}$ 利用非 离子型表面活性剂壬基酚聚氧乙烯(10)醚(PONPE10)以 及配体 2-乙基己基磷酸-2-乙基己基酯(EHPNA)分离 $\mathrm{Co}^{2+}$ 和 $\mathrm{Ni}^{2+}$, 当 PONPE10 和 EHPNA 浓度分别为 0.05 和 $0.002 \mathrm{~mol} \cdot \mathrm{L}^{-1}$ 时, 分离系数可以达到 6.4. 同时含有 $\mathrm{Pb}^{2+}$ 和 $\mathrm{AsO}_{4}^{-}$的混合废水用 MEUF 回收金属离子, 发现 当阴离子表面活性剂苯磺酸(DSA)和阳离子表面活性剂 十二胺的浓度分别为 $10^{-5}$ 和 $10^{-6} \mathrm{~mol} \cdot \mathrm{L}^{-1}$ 时, 对于 $\mathrm{Pb}^{2+}$ 和 $\mathrm{AsO}_{4}^{-}$的截留率可以达到 $99.0 \%$ 和 $80.0 \%{ }^{[51]}$. Huang 等 ${ }^{[52]}$ 研究发现阴离子/非离子表面活性剂混合体系 SDS/ 聚氧乙烯辛基苯基醚 Triton X-100 对于 $\mathrm{Cd}^{2+}$ 的截留率可 以达到 $98.7 \%$, 与单独 SDS 体系相比, 表面活性剂用量 降低了 $50 \%$, SDS 的残留率降低约 $25 \%$. 对于带负电荷 的无机阴离子, 例如, $\mathrm{NO}_{3}^{-[53]}, \mathrm{CrO}_{4}^{2-}{ }^{[54 \sim 56]}, \mathrm{AsO}_{4}^{3-}$ [57] 和 $\mathrm{Fe}(\mathrm{CN})_{6}^{3-}{ }^{[58]}$, 通常选取阳离子表面活性剂 $\mathrm{CPC}$ 及

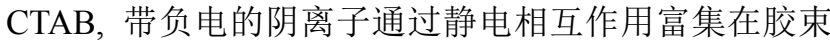
表面从而被有效去除.

胶束增强超滤技术也可以用于去除废水中的有机 污染物，如烷烃、胺、醇、芳香烃、酚和氯代芳烃化合 物等, 截留率都在 $80 \%$ 以上(表 1$)^{[59 \sim 62]}$. 其原理是表面 活性剂形成的胶束能够增溶有机物, 截留率取决于有机 物自身的结构和性质以及表面活性剂胶束对于有机污 染物的增溶能力. 从表 1 中可以看出，随着烷基链增长, 疏水性增加, MEUF 对于正己醇、正庚醇和正辛醇的截 留率逐渐增加, 这是由于表面活性剂形成的胶束对于有 机物的增溶能力随着有机物疏水性增加而增大. 疏水性 更强的甲苯的截留率高于苯. 苯酚在水中电离后带负电 荷, 因此, 使用阳离子表面活性剂 CPC 和阴离子表面活 性剂 SDS 去除苯酚时, 苯酚更易于和带正电荷的胶束 结合, 截留率更高. 利用胶束增强超滤技术可以去除染 料废水中的染料分子. 2004 年, Purkait 等 ${ }^{[63,64]}$ 首次利用 阳离子表面活性剂 CPC 实现了对于水中阴离子型染料 曙红的提取. 随着表面活性剂浓度增加, 曙红的截留率 逐渐增加, 与不添加表面活性剂的实验条件相比, 截留 率从 $10 \%$ 增加至 $73 \%$. 对于带电荷的染料分子, 通常选 取带有相反电荷的表面活性剂，二者可以通过静电相互 作用形成离子对，例如 SDS 对于阳离子型染料亚甲基 蓝的截留率为 $94 \%$, 而非离子型表面活性剂 OMC-10 由 于不能与亚甲基蓝分子形成离子对，截留率只有 $50 \%$ [37,60,65].

表 1 MEUF 对有机物的去除效果 ${ }^{[59 \sim 62]}$

Table 1 Removal efficiency of organic compounds by MEUF technique ${ }^{[59 \sim 62]}$

\begin{tabular}{lccc}
\hline \multicolumn{1}{c}{ 有机物 } & 有机物浓度 $/\left(\mathrm{mmol} \cdot \mathrm{L}^{-1}\right)$ & 表面活性剂 & 截留率 $/ \%$ \\
\hline 苯酚 & 1.42 & $\mathrm{CPC}$ & 92.9 \\
& 1.00 & $\mathrm{SDS}$ & 33.1 \\
苯 & 2.33 & $\mathrm{CPC}+\mathrm{CTAB}$ & 88.4 \\
甲苯 & 0.80 & $\mathrm{CPC}$ & 96.0 \\
正己烷 & 0.05 & $\mathrm{CPC}$ & 99.8 \\
正己醇 & 1.56 & $\mathrm{CPC}$ & 92.2 \\
正辛醇 & 0.14 & $\mathrm{CPC}$ & 99.3 \\
正庚醇 & 0.32 & $\mathrm{CPC}$ & 98.4 \\
4-叔丁基酚 & 0.07 & $\mathrm{CPC}$ & 99.6 \\
铬黑 & 0.11 & $\mathrm{CTAB}$ & 99.3 \\
& 0.11 & $\mathrm{CTAB}+\mathrm{OMC}-10$ & 98.6 \\
亚甲基蓝 & 0.02 & $\mathrm{SDS}$ & 99.3 \\
番红精 $\mathrm{T}$ & 1.00 & $\mathrm{SDS}$ & 99.0 \\
\hline
\end{tabular}

\section{3 固相吸附法}

固相吸附法是利用多孔固体材料除去废水中金属 离子和有机物的一种方法. 与传统液相萃取法相比, 固 相吸附法操作简便, 提取效率高, 主要用于环境监测和 样品预处理. 对于固体吸附剂的改性, 例如沸石、活性 
炭等, 能够提高固体吸附剂的比表面积和阳离子交换 量, 改善固体吸附剂对于金属离子、无机阴离子以及有 机污染物的吸附能力, 从而提高其使用价值. 常见的固 相改性方法包括热改性、酸改性、碱改性、无机改性、 有机改性等方法. 利用表面活性剂对于固相吸附剂进行 改性是固相吸附剂有机改性的主要途径. 表面活性剂吸 附在固定相表面, 可以形成半胶团(hemimicelle). 这种 结构与水溶液中的胶束类似, 能够增溶废水中的有机物 或吸附金属离子, 提高固定相的吸附能力. 被吸附物质 在溶液和固相半胶团之间的分配过程为增溶过程.

\section{1 表面活性剂在固体表面的吸附}

由于硅酸盐、金属氧化物以及一些纤维的表面都是 带有负电荷的, 对于吸附污染物的固相改性时一般选取 阳离子表面活性剂, 最常用的是季铵盐型阳离子表面活 性剂.

离子型表面活性剂在金属氧化物固相表面的吸附 符合经典的 $\mathrm{S}$ 型吸附过程(图 2) ${ }^{[46]}$, 吸附等温线被划分 为四个区域: 在区间 I, 表面活性剂分子以单体的形式 通过静电相互作用与固体表面结合, 吸附量与固体表面 电荷量有关, 被吸附的表面活性剂分子之间的相互作用 可以忽略不计，此区间也被称作 Henry’s Law 区间; 在 区间 II, 表面活性剂分子通过与已被吸附的表面活性剂 分子的协同作用吸附在固体表面，吸附量增加; 在区间 III, 此时表面活性剂浓度达到半胶束化浓度, 即 $\mathrm{hmc}$ (the hemimicelle concentration), 表面活性剂分子通过疏 水相互作用在固相表面形成球形或者双层结构, 此时固 体表面电荷被中和, 电性发生反转; 在区间 IV, 当浓度 达到临界胶束浓度 $\mathrm{CMC}$ 时, 表面活性剂分子在溶液中 形成胶束, 在固体表面的吸附量不再发生变化. 表面活 性剂分子在固体表面的吸附受多种因素的影响, 例如无 机盐和有机盐加入、表面活性剂结构的变化、 $\mathrm{pH}$ 值以 及温度的改变等. 表面活性剂改性固相表面形成的双分 子层具有增溶作用, 被用于提取金属离子、药物分子和 土壤修复等多方面.
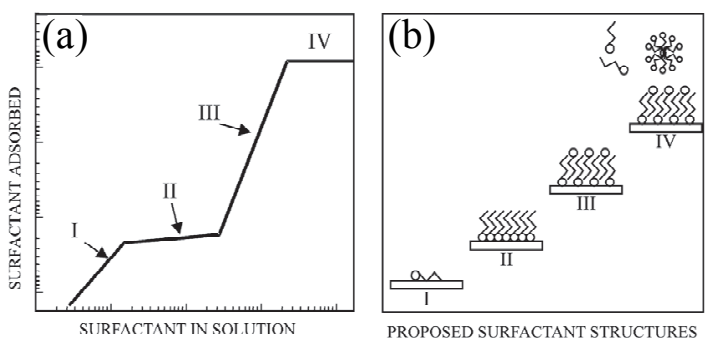

图 2 离子型表面活性剂在固体表面的吸附等温线(a)及示意图(b ${ }^{[66]}$ Figure 2 Adsorption isotherms of ionic surfactants on solid surfaces (a) and structures proposed for the surfactant adsorbed in each region $(b)^{[66]}$

\section{2 固相吸附法用于去除水中污染物}

在废水处理应用中, 常用的表面活性剂改性的固定 相包括活性炭、沸石、硅胶和黏土 ${ }^{[67 ~ 71]}$.
活性炭是废水处理中常用的固相吸附剂，具有复杂 的孔状结构，比表面积大，化学稳定性好，对于非极性 的分子吸附能力较强, 表面的含氧官能团和杂原子使其 对极性分子和离子也有一定的吸附能力, 但吸附能力较 弱, 属于疏水型非极性吸附剂. 离子型表面活性剂改性 后的活性炭, 表面活性剂分子通过疏水相互作用或静电 相互作用与固体表面结合，改性后的固定相表面电荷量 增加, 可以通过离子交换或静电相互作用结合更多离 子，提高了活性炭对金属离子或极性有机物的吸附量. Farooq 等 ${ }^{[73]}$ 利用阳离子表面活性 CTAB, 十六烷基三甲 基氯化铵(CTAC)和 CPC 改性后的粉末活性炭去除水中 的溴酸盐 $\left(\mathrm{BrO}_{3}^{-}\right)$, 发现 CTAC 和 CPC 改性后的粉末活 性炭通过离子交换作用去除 $\mathrm{BrO}_{3}^{-}$, 其吸附速率及吸附 量均高于未改性的活性炭, 具有较好的去除效果. 当溶 液 $\mathrm{pH}$ 值小于 7 时, $\mathrm{BrO}_{3}^{-}$通过静电相互作用被吸附在改 性活性炭的孔道中; 当 $\mathrm{pH}$ 值继续增大, 由于电荷性质 的反转, 改性活性炭对于 $\mathrm{BrO}_{3}^{-}$的提取效率降低. Chen 等 ${ }^{[74]}$ 也证明了 $\mathrm{CTAC}$ 改性的活性炭对于 $\mathrm{BrO}_{3}^{-}$的吸附能 力可以达到 $30.30 \mathrm{mg} / \mathrm{g}$, 而未改性活性炭的吸附能力只 有 $5.53 \mathrm{mg} / \mathrm{g}$. Hong 等 ${ }^{[75]}$ 发现 CPC 改性的活性炭联合超 滤的方法对于水中铬酸盐 $\left(\mathrm{CrO}_{4}^{2-}\right)$ 、砷酸盐 $\left(\mathrm{AsO}_{4}^{3-}\right)$ 和铁 氰化物 $\left[\mathrm{Fe}(\mathrm{CN})_{6}^{3-}\right]$ 具有较好的去除效果, 最大吸附量分 别为 $0.234,0.087$ 和 $0.085 \mathrm{mmol} / \mathrm{g}$. 阴离子表面活性剂可 以通过疏水相互作用与活性炭的非极性部位结合，极性 头基朝向水相，使得活性炭电荷带有更多负电荷，提高 了其对于正电荷金属离子的吸附能力. Ahn 等 ${ }^{[72]}$ 利用 SDS、十二烷基苯磺酸钠(SDBS)和丁二酸二辛酯磺酸钠 (DSS)改性的活性炭提取水中的 $\mathrm{Cr}^{2+}$, 最大吸附量可以 达到 $0.198 \mathrm{mmol} / \mathrm{g}$ (图 3). 阴离子表面活性剂二乙基二硫 代氨基甲酸钠(SDDC)改性的固定床活性炭柱对于 $\mathrm{Cu}^{2+}$ 和 $\mathrm{Zn}^{2+}$ 的吸附能力是未改性活性炭柱的四倍, 最大吸 附量分别为 38 和 $9.9 \mathrm{mg} / \mathrm{g}^{[76]}$. 此外, 离子型表面活性剂 改性的活性炭对染料分子也具有更强的吸附能力, 例如 亚甲基蓝、活性黑 5、酸性红和酸性蓝等 ${ }^{[77 ~ 80]}$.
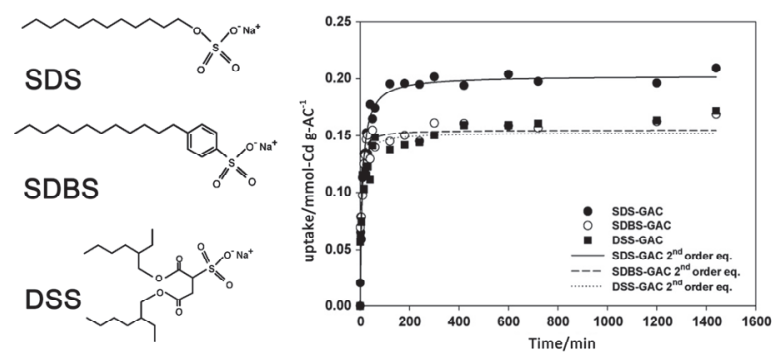

图 $3 \mathrm{SDS}$ 、 SDBS 以及 DSS 改性的活性炭对 $\mathrm{Cd}^{2+}$ 的吸附量与时间的 关系以及相应的二级动力学拟合曲线 ${ }^{[72]}$

Figure $3 \mathrm{Cd}^{2+}$ uptake during adsorption onto SDS, SDBS and DSS modified activated carbon with pseudo-second-order kinetic model fitting $^{[72]}$

沸石是富含钾、钠、钙、钡离子的硅铝酸盐矿物，具 有孔道结构，能够通过阳离子的离子交换实现对于金属 
离子的吸附. 由于表面硅氧基团的亲水性，沸石对于阴 离子型污染物以及有机物的吸附性能较差. 表面活性剂 改性的沸石不仅提高了对于无机阴离子如硫酸盐、高氯 酸盐的吸附能力, 还能够通过静电相互作用和疏水相互 作用高效地增溶有机污染物. 阳离子表面活性剂是常用 的沸石有机改性剂, 包括四乙基溴化铵(TEA)、十六烷 基三甲基溴化铵(CTAB)、十六烷基溴化吡啶(CPB)及 $N-$ 苄基- $N, N$-二甲基十八烷基-1-氯化铵(SDBAC)等. 表面 活性剂在沸石表面形成了紧密排布的双层结构，提高了 沸石对于无机酸根离子的吸附能力. Ghiaci ${ }^{[82]}$ 和 Bowman 等 ${ }^{[83]}$ 应用 CTAB 和 CTAC 改性后的沸石吸附废 水中的铬酸盐 $\left(\mathrm{CrO}_{4}^{2-}\right)$, 发现改性沸石对于 $\mathrm{CrO}_{4}^{2-}$ 的去 除效率达到 $90 \%$ 以上. Macedo-Miranda 和 Olguín ${ }^{[84]}$ 发 现 CTAB 改性的沸石提高了沸石对于 $\mathrm{AsO}_{4}^{3-}$ 的吸附能 力, 吸附效率与溶液 $\mathrm{pH}$ 值、砷元素价态以及沸石的结 构特点有关. Chutia 等 ${ }^{[85]}$ 发现 CTAB 改性的丝光沸石和 天然斜发沸石对于 $\mathrm{AsO}_{4}^{3-}$ 的吸附量分别为 97.33 和 45.33 $\mathrm{mmol} / \mathrm{kg}$. 阳离子表面活性剂改性的沸石也被用于吸附

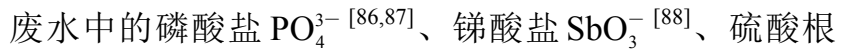
$\mathrm{SO}_{4}^{2-}$ 和硒酸根 $\mathrm{SeO}_{4}^{2-}$ [89]等. 改性后的沸石可以通过与 沸石中残留的阳离子的离子交换吸附金属离子. Liu 等 ${ }^{[90]}$ 利用 CPB 对沸石进行改性, 发现改性后的沸石对 于 $\mathrm{Hg}^{2+}$ 的饱和吸附量是未改性沸石的 16.35 倍. 吸附机 理包括疏水相互作用、离子交换、表面吸附和电荷中和 等多种作用, 竞争离子的存在对于吸附效果影响较小. Cortés-Martínez 等 ${ }^{[91]}$ 研究发现 CTAB 改性的沸石对于 $\mathrm{Cd}^{2+}$ 的吸附能力稍低于天然沸石对于 $\mathrm{Cd}^{2+}$ 的吸附能力, 但是在阴离子和非极性有机物存在时具有明显的优势. 表面活性剂的改性能够提高沸石对于有机物的吸附能 力. Torabian 等 ${ }^{[92]}$ 研究了 CTAC 和 CPB 改性后的沸石对 于苯、甲苯、二甲苯和乙苯的吸附效果, 发现随着表面 活性剂的浓度从低于 $\mathrm{CMC}$ 值提高至高于 $\mathrm{CMC}$ 值, 吸附 效率分别可以达到 70\%和 $99 \%, \mathrm{CPB}$ 改性沸石对于苯系 物的吸附效果优于 CTAC 改性沸石. Dong 等 ${ }^{[81]}$ 发现 CTAB 改性的沸石对于双酚 A 具有优异的吸附能力, 提 高溶液 $\mathrm{pH}$ 值和离子强度以及降低温度能够提高改性沸 石对双酚 $\mathrm{A}$ 的提取效率. 不带电荷的双酚 $\mathrm{A}$ 可以通过疏 水相互作用以及氧原子给电子作用被吸附在沸石表面, 而阴离子型的双酚 $\mathrm{A}$ 则通过静电相互作用和疏水相互 作用与表面结合 (图 4). Abatal 等 ${ }^{[93]}$ 研究了 CTAB 改性沸 石对于苯酚和对硝基苯酚的吸附效果, 发现改性沸石能 实现对酚类有机物的快速吸附, 而且改性沸石对于对硝 基苯酚的去除效果优于苯酚. 利用十二烷基三甲基溴化 铵(DTAB)改性的沸石去除腐殖酸时, 腐殖酸分子通过 疏水相互作用和氢键被吸附在固体表面, 表面活性剂单 分子层最利于腐殖酸的吸附 ${ }^{[94]}$. Benkli 等 ${ }^{[95]}$ 研究了 CTAB 改性沸石对于三种活性染料 CI Reactive Black 5, Red 239 和 Yellow 17 的吸附效果, 发现改性沸石对于 CI Reactive Black 5 的吸附效果最好, 且表面活性剂双
分子层结构有利于阴离子型染料与表面活性剂的相互 作用. 表面活性剂改性沸石也被用于提取废水中的农 药 ${ }^{[96]}$ 和微生物 ${ }^{[97]}$ 等. 此外, 表面活性剂改性沸石与其他 改性方式联用，例如，碱改性、配体改性及稀土改性等， 可以提高沸石对于污染物的吸附能力，能够实现对于金 属离子、含氧阴离子及有机化合物等多种污染物的高效 吸附 ${ }^{[98 \sim 100]}$.
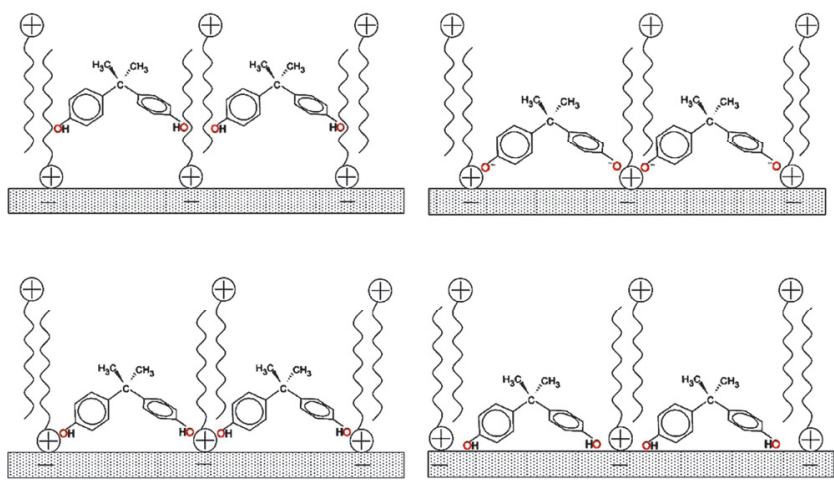

图 4 CTAB 改性沸石与 BPA 的作用机理 ${ }^{[81]}$

Figure 4 Interaction of $\mathrm{CTAB}$ modified zeolite with $\mathrm{BPA}^{[81]}$

黏土类矿物质也是一类吸附能力较强的硅铝酸盐 矿物质，根据层结构的不同可以分为蒙脱土、云母、高 岭土、海泡石等不同类型. 黏土类物质比表面积较大， 具有丰富的孔道结构，可以通过表面吸附、离子交换等 方式实现对废水中金属离子的吸附. 黏土类物质层间被 无机阳离子 $\left(\mathrm{Ca}^{2+}, \mathrm{Na}^{+}, \mathrm{K}^{+}\right)$和水分子占据, 呈现亲水性, 对于有机污染物的吸附效果较差, 特别是弱极性或非极 性的有机物. 利用表面活性剂对黏土类矿物质进行有机 改性，使其表面转变为疏水性表面，有利于提高其对废 水中污染物的吸附效果, 扩大应用范围. 以蒙脱土(膨 润土)为例，离子表面活性剂分子可以通过离子交换以 单层平铺、双层平铺、假三层、单层倾斜或双层倾斜等 方式插入蒙脱土层状结构中，使层间距增大，蒙脱土表 面以及层间疏水性增加, 有效地提高了对于有机物的吸 附效率. 层间距越大, 蒙脱土(膨润土)的疏水性越好, 对于有机污染物的吸附能力越强. 改性后的黏土类矿物 的结构性能与表面活性剂的结构、浓度以及配比有关. 调节表面活性剂的配比能够得到不同层间距的黏土，进 而实现对于黏土结构和性能的有效调控.

自 1949 年 Jordan ${ }^{[102]}$ 首次利用阳离子季铵盐型表面 活性剂改性蒙脱土(膨润土), 国内外学者对于表面活性 剂改性蒙脱土(膨润土)的吸附有机污染物的性能、机理 及废水处理应用方面做了大量的研究工作. 1995 年, Smith 等 ${ }^{[103]}$ 利用不同碳链长度的阳离子季铵盐型表面活 性剂改性膨润土; Shen ${ }^{[104]}$ 制备了非离子表面活性剂改性 的膨润土; 朱利中等 ${ }^{[105]}$ 利用 SDS 和 CTAB 阴阳离子表 面活性剂混合体系改性膨润土，此外，还制备了双阳离 子表面活性剂改性有机膨润土 ${ }^{[106]}$ 和阳离子/非离子有机 
膨润土 ${ }^{[107]}$, 提高了膨润土对于有机污染物的吸附能力. 朱利中课题组 ${ }^{[107]}$ 系统地研究了阳离子表面活性剂改性 的膨润土对于废水中有机物的吸附性能及机理, 发现改 性膨润土对于有机物的吸附能力与表面活性剂的链长、 化学结构以及膨润土的阳离子交换量有关. 该课题组发 明了有机膨润土合成一废水吸附处理一体化工艺，向废 水中同时加入少量表面活性剂和膨润土, 同步实现有机 膨润土自组装和废水的吸附处理, 该工艺可以高效去除 有机污染物, 简化工艺流程, 改善废水可生化性, 特别 适合中高浓度难降解有机废水的预处理, 并应用到实际 废水处理工程, 取得了很好的效果 ${ }^{[108,109]}$. Liu 等 ${ }^{[101]}$ 利用 十八烷基二甲基甜菜碱改性的蒙脱土处理同时含有双酚 $\mathrm{A}$ 和 $\mathrm{Cd}^{2+}$ 的水溶液中, 取得了良好的吸附效果. $\mathrm{Cd}^{2+}$ 通 过静电相互作用以及配位作用与固体表面结合, 而双酚 A 则主要通过疏水相互作用被固定相吸附(图 5). Nourmoradi 等 ${ }^{[110]}$ 发现十四烷基三甲基溴化铵 TTAB 改性后 的蒙脱土对于苯、甲苯、乙苯和二甲苯的吸附速率明显 增大, 最大吸附量分别为 $3.98,5.15,6.00$ 和 $6.98 \mathrm{mg} / \mathrm{g}$. 表面活性剂改性的蒙脱土还被应用于吸附废水中的染料

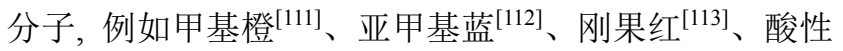
红 ${ }^{[114]}$ 等. 表面活性剂改性的蒙脱土可以用于去除废水 中的重金属离子. Lin 等 ${ }^{[115]}$ 对比了 SDS 改性蒙脱土和未 改性蒙脱土对于 $\mathrm{Cu}^{2+}$ 和 $\mathrm{Zn}^{2+}$ 的吸附量, 结果表明, 改性 蒙脱土对于金属离子的吸附能力明显优于未改性蒙脱 土, 且对于 $\mathrm{Cu}^{2+}$ 的吸附量大于 $\mathrm{Zn}^{2+}$, 吸附等温线符合 Langmuir 模型. Malakul 等 ${ }^{[116]}$ 选取了阴阳离子表面活性 剂体系 $N$-十六烷基- $N, N$-二甲基芳基氯化铵(CDBA)和十 六酸 PA 改性蒙脱土, 发现改性后蒙脱土对于 $\mathrm{Cd}^{2+}$ 的吸 附量可以达到 $(42 \pm 0.8) \mathrm{mg} / \mathrm{g}$. 此外, $\mathrm{Li}^{[117]}$ 和 Özcan 等 ${ }^{[118]}$ 还研究了表面活性剂改性蒙脱土对于 $\mathrm{Co}^{2+}$ 和 $\mathrm{Fe}^{2+}$ 的吸 附. 朱利中等 ${ }^{[119,120]}$ 利用阳离子表面活性剂和铝或铁盐 同时改性制备有机-无机改性膨润土, 可以同时去除废 水中的有机污染物和磷酸盐, 提取效率均在 $90 \%$ 以上.

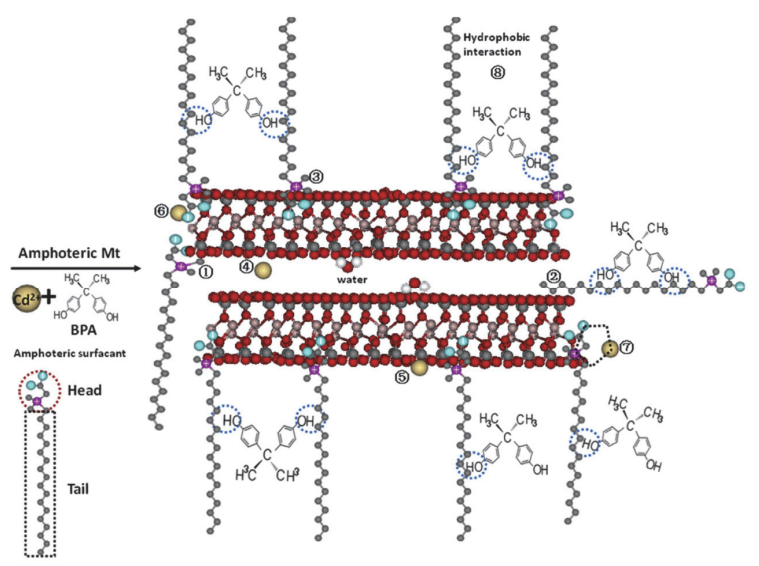

图 5 十八烷基二甲基甜菜碱改性蒙脱土对于 $\mathrm{Cd}^{2+} / \mathrm{BPA}$ 的吸附机理 ${ }^{[101]}$ Figure 5 Addsorption mechanism of $\mathrm{Cd}^{2+}$ and BPA onto octadecane-betaine modified montmorillonite ${ }^{[101]}$

\section{4 基于液液相分离的提取方法}

\section{1 液液相分离原理}

20 世纪 30 年代, Bungenberg de Jong 等 ${ }^{[121]}$ 研究了阿 拉伯胶一明胶体系, 并首次提出了液液相分离的概念. 液液相分离是指均一的胶体物质或大分子水溶液自发 分为两个互不相溶的液相的过程, 其中, 富含胶体物质 或大分子的一相为凝聚相, 另一相为稀溶液相 (图 $6)^{[122 \sim 129]}$.

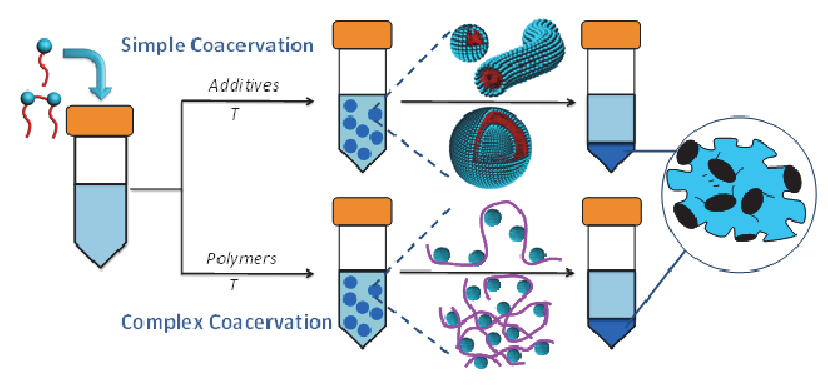

图 6 液液相分离的分类及作用机理 ${ }^{[128]}$

Figure 6 Classification and mechanism of coacervation systems ${ }^{[128]}$

液液相分离可以分为两类: 简单的液液相分离体系 和复杂的液液相分离体系. 简单液液相分离体系是指单 一的表面活性剂或大分子溶液，通过升高温度或加入无 机盐、有机盐、醇等脱水剂促进表面活性剂或大分子之 间的相互作用实现相分离. 烷基聚氧乙烯类非离子表面 活性剂 $\left(\mathrm{C}_{\mathrm{i}} \mathrm{E}_{\mathrm{j}}\right)$ 的水溶液, 升高体系温度至大于临界温度 时，胶束之间作用力增强，体系发生液液相分离，这一 现象也被称作浊点现象，相应的临界温度被称为浊

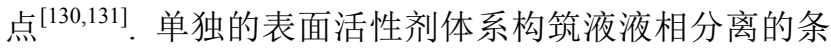
件较苛刻, 对于大部分表面活性剂体系, 需要加入助剂, 调节分子间相互作用来构筑液液相分离. 无机盐的加入 能够屏蔽离子型表面活性剂头基之间的静电排斥作用, 诱导聚集体增长, 从而发生液液相分离. Cohen 等 ${ }^{[132 ~ 137] ~}$ 研究了无机盐诱导的阳离子季铵盐表面活性剂 Hyamine 1622 的液液相分离体系, 实验结果表明, 当无机盐浓度 超过临界值时, 由于屏蔽作用, 胶束之间的静电斥力减 弱, 球形胶束逐渐增长成为柱状胶束前驱体，体系最终 发生液液相分离. 有机盐不仅可以屏蔽表面活性剂分子 间的静电排斥作用, 还可以通过增强疏水相互作用以及 氢键作用调节体系中分子间作用力, 实现液液相分离. 我们课题组 ${ }^{[138]}$ 利用 Gemini 表面活性剂 1,6-双 $(N$-十二烷 基- $N, N$-二甲基溴化铵)-己烷(12-6-12)与 $N$-苯甲酰基谷 氨酸 $\left(\mathrm{H}_{2} \mathrm{Bzglu}\right)$ 通过静电相互作用、疏水相互作用和氢键 构筑了液液相分离. 醇类物质可以诱导单链的非离子表 面活性剂、两性离子表面活性剂以及离子表面活性剂体 系液液相分离的形成 ${ }^{[139,140]}$. 酸的加入可以使阴离子表 面活性剂十二烷基硫酸钠、十二烷基苯磺酸钠及十二烷 基磺酸钠的硫酸基团和磺酸基团质子化，静电排斥作用 
减小, 聚集体间相互作用增强, 形成液液相分离 ${ }^{[141,142]}$.

复杂液液相分离体系包含至少两种胶体物质, 通过 调节分子间的相互作用或环境因素实现相分离. 目前, 关于液液相分离的文献报道主要集中于由大分子形成 的复杂液液相分离体系, Dubin ${ }^{[124,143 \sim 146]}$ 、Veis ${ }^{[123]}$ 和 Bohidar 等 ${ }^{[122]}$ 在这一方面做出了突出的贡献. 相比较而 言, 基于表面活性剂的复杂液液相分离体系的文献报道 较少, 主要包括非离子型表面活性剂/非离子型聚合物 液液相分离、非离子型表面活性剂/聚电解质液液相分 离、离子型表面活性剂/非离子型聚合物液液相分离以及 带相反电荷的离子型表面活性剂/聚电解质液液相分离. 非离子型表面活性剂或非离子型聚合物参与形成的复 杂液液相分离, 例如 $\mathrm{C}_{12} \mathrm{E}_{6} /(\mathrm{PEO})_{20}(\mathrm{PPO})_{68}(\mathrm{PEO})_{20^{-}}$ $(\mathrm{P} 105)^{[147]}$, 通常需要升高体系温度来实现, 这一点与单 一非离子表面活性剂体系的浊点现象类似. 非离子型表 面活性剂/聚电解质混合体系和离子型表面活性剂/非离 子型聚合物混合体系在构筑液液相分离时, 通常需要加 入无机盐用于屏蔽表面活性剂带电头基或聚电解质带 电结构单元之间的静电乕力 ${ }^{[148,149]}$. 对于带相反电荷的 表面活性剂/聚电解质液液相分离体系, 表面活性剂的 临界聚集浓度 CAC 小于表面活性剂自身的 CMC 值; 当 浓度高于 $\mathrm{CAC}$ 时, 表面活性剂形成较低聚集数的聚集 体, 在溶液中二者复合形成珍珠项链结构; 复合物之间 相互交联, 体系发生液液相分离; 进一步增加表面活性 剂的浓度, 凝聚相被过量的表面活性剂胶束溶解 ${ }^{[150,151]}$.

\section{2 液液相分离提取技术}

液液相分离提取技术是利用表面活性剂在一定条 件下发生液液相分离, 液液相分离形成的凝聚相富含大 量表面活性剂分子, 具有三维网状结构, 可以增溶溶液 中的污染物, 从而实现对于污染物的富集和分离. 其中 最具有代表性的是浊点提取法 CPE (Cloud Point Extraction), 它利用的是非离子表面活性剂的浊点现象. 20 世 纪 70 年代, Watanabe 等 ${ }^{[152]}$ 首次报道了利用浊点现象提 取水溶液中的金属离子, 之后该技术迅速发展, 被广泛 地应用在有机物、金属离子以及蛋白质的分离提取过 程 ${ }^{[63,153]}$. 近二十年, 基于离子型表面活性剂的液液相分 离提取技术逐渐发展, 扩大了液液相分离体系的应用范 围.

典型的液液相分离技术包括以下几个步骤: (1)增 溶: 将表面活性剂添加到含有被提取物的溶液中, 混合 均匀后, 污染物被增溶到表面活性剂形成的胶束中; (2) 相分离: 通过添加其他助剂, 改变温度或调节 $\mathrm{pH}$ 等条 件实现液液相分离; (3)凝聚相回收: 凝聚相和水相通过 沉降或离心等方式实现两相彻底分离, 实现凝聚相的回 收, 用水或有机溶剂对凝聚相进行稀释, 进行下一步的 定性或定量分析. 与传统分离技术相比, 液液相分离提 取技术具有以下特点: (1)表面活性剂分子可以通过自然 界或化学合成得到, 成本较低; (2)调节表面活性剂分子
的疏水尾链或极性头基可以实现对不同极性的化合物 的提取; (3)减少有机溶剂的使用, 更加环保、安全; (4) 表面活性剂形成的凝聚相具有非挥发性和非可燃性; (5) 对于被提取物质具有较高的富集效率 ${ }^{[154]}$. 因此, 液液 相分离提取技术为废水处理提供了新的选择, 成为科学 家研究的热点问题.

\section{3 液液相分离提取技术用于去除水中污染物}

液液相分离体系在废水处理应用的文献中，对于浊 点提取法的报道最为广泛、深入. 利用浊点提取法进行 分离提取时, 溶液温度必须高于浊点温度. 降低非离子 表面活性剂的浊点温度能够有效降低废水处理过程的 能耗. 非离子表面活性剂的浊点主要受两个因素的影 响: (1)结构因素: 延长疏水尾链链长、降低 $\mathrm{EO}$ 基团的数 目或选取分子量分布更宽的表面活性剂分子都能够有 效地降低非离子表面活性剂的浊点; (2)添加剂的影响: 向表面活性剂溶液中加入氯化物、硫酸盐和碳酸盐时, 由于盐析效应会使浊点降低. 另一方面, 硫氰酸盐、碘 化物和硝酸盐等由于盐溶效应会使浊点增加. 饱和烃类 能够引起浊点降低, 离子型表面活性剂的添加使浊点增 大. 因此，调整表面活性剂分子的结构或加入合适的无 机盐能够有效降低非离子表面活性剂的浊点 ${ }^{[23]}$.

浊点提取法被广泛地应用于多种物质的提取和分

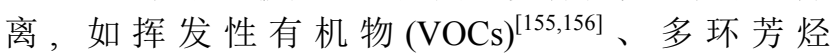

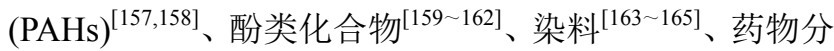
子[166,167]和金属离子 ${ }^{[168 ~ 176]}$ 等. 表 2 给出了 $\mathrm{CPE}$ 提取有 机物及金属离子的总结. 最常用的非离子表面活性剂为 聚氧乙烯辛基苯基醚 Triton X-114、Triton X-100 和 $\alpha$ 异十三烷基- $\omega$-羟基-聚(氧-1,2-亚乙基)Genapol X-080, 选取的表面活性剂浓度接近 $0.01 \mathrm{~g} / \mathrm{mL}$. 在实际操作过 程中, 通常会加入 $\mathrm{Na}_{2} \mathrm{SO}_{4},\left(\mathrm{NH}_{4}\right)_{2} \mathrm{SO}_{4}$ 或 $\mathrm{NaCl}$ 使浊点温 度在 $35 \sim 70{ }^{\circ} \mathrm{C}$ 范围内. Trakultamupatam 等 ${ }^{[177]}$ 利用 $\mathrm{OP}(\mathrm{EO})_{7}$ 提取苯、甲苯和乙苯, 发现表面活性剂浓度为 $100 \mathrm{mmol} \cdot \mathrm{L}^{-1}$ 时，提取效率接近 $85 \%$. 提高体系的操作 温度、无机盐浓度或增加有机物的烷基化程度，能够增 强胶束之间的相互作用，使得凝聚相的堆积更加紧密， 有利于有机物的富集. 此外，表面活性剂的结构也会影 响有机物在两相中的分配. Taechangam 等 ${ }^{[159]}$ 研究发现 苯酚在非离子表面活性剂形成的凝聚相中的分配随着 EO 数量线性增加, 但不受碳链长度或支化度的影响. 这是由于苯酚通过羟基与栅栏层中 EO 基团的偶极-偶 极相互作用被增溶在胶束表面, $\mathrm{EO}$ 基团越多，与 $\mathrm{OH}$ 基 团的相互作用越强, 苯酚在凝聚相中的分配系数越高. 在对于金属离子的分析中, CPE 通常作为痕量物质检测 的预浓缩萃取手段，与原子吸收光谱仪(AAS)、电感耦 合等离子体光谱仪(ICP)等设备相联. 金属离子可以通 过与表面活性剂头基的静电相互作用与胶束结合，或与 添加的配体络合后进一步被胶束增溶.

非离子表面活性剂/离子表面活性剂的混合液液相 
分离体系同时利用静电及疏水相互作用, 有利于提高对 带电荷的有机物的提取效率. Pourreza 等 ${ }^{[178]}$ 研究发现 CTAB/Triton X-100 混合体系升温形成的凝聚相对于阴 离子染料 Orange II 的提取效率可达到 $99.8 \%$, 这是由于 Orange II 为阴离子型染料, 可以通过静电相互作用与 CTAB 结合, 有利于富集在凝聚相中. 提高表面活性剂 的浓度或加入一定浓度的无机盐能够提高凝聚相对于 Orange II 的提取效率. 此外, 文献报道 CTAB/Triton X-114 混合体系可以提取水溶液中的 Humic Acid, Fulvic Acid $^{[179]}$ 和青需素 ${ }^{[180]}$. Zarei 等 ${ }^{[181]}$ 利用阴离子表面活性 剂 SDS/Triton X-114 混合体系形成的凝聚相提取酰肼. 酰肼和添加剂对二甲氨基苯甲醛 DAB 反应形成带正电 荷的鲐嗪, 与 SDS 通过静电相互作用结合, 提高了反应 的速率和平衡常数, 进一步促进了浐嗪的富集过程.

表 $2 \mathrm{CPE}$ 提取有机物及金属离子汇总 ${ }^{[154,168 ~ 175]}$

Table 2 Summary of organic compounds and metal ions extracted by CPE method ${ }^{[154,168 \sim 175]}$

\begin{tabular}{|c|c|c|c|}
\hline 被提取物 & 表面活性剂 & 条件 & 提取效率/\% \\
\hline $\begin{array}{l}\text { 菲屈 } \\
\text { 苯并芘 } \\
\text { 苯并菼蒽 }\end{array}$ & Tergitol 15-S-7 & $1.5 \mathrm{~mol} \cdot \mathrm{L}^{-1}\left(\mathrm{NH}_{4}\right)_{2} \mathrm{SO}_{4}$ & $88 \sim 105$ \\
\hline 苯并萠 & Triton X-114 & $0.6 \mathrm{~mol} \cdot \mathrm{L}^{-1} \mathrm{NaSO}_{4}$ & 98 \\
\hline 多环芳烃 & PEG-10-lauryl ether & $90{ }^{\circ} \mathrm{C}$ & $38 \sim 104$ \\
\hline 扑草净 & Triton X-114 & $0.03 \mathrm{~g} / \mathrm{mL} \mathrm{NaCl}$ & $93 \sim 99$ \\
\hline 罗丹明 B & Triton X-100 & $78{ }^{\circ} \mathrm{C}, 0.1 \mathrm{~mol} \cdot \mathrm{L}^{-1} \mathrm{NaCl}$ & $96 \sim 103$ \\
\hline 苏丹染料 & Triton X-100 & $70{ }^{\circ} \mathrm{C}, 0.1 \mathrm{~g} / \mathrm{mL} \mathrm{Na}_{2} \mathrm{CO}_{3}$ & $81 \sim 85$ \\
\hline 雌三醇 & Triton X-114 & $45{ }^{\circ} \mathrm{C}, 0.4 \mathrm{~mol} \cdot \mathrm{L}^{-1} \mathrm{Na}_{2} \mathrm{SO}_{4}$ & $81 \sim 99$ \\
\hline \multicolumn{4}{|l|}{ 2,4-硝基苯酚 } \\
\hline \multicolumn{4}{|l|}{ 2-硝基苯酚 } \\
\hline 2-氯苯酚 & Genapol X-080 & $85{ }^{\circ} \mathrm{C}, 0.06 \mathrm{~g} / \mathrm{mL} \mathrm{NaCl}$ & $95 \sim 114$ \\
\hline \multicolumn{4}{|l|}{ 2,4-二甲基苯酚 } \\
\hline \multicolumn{4}{|l|}{ 对甲基苯酚 } \\
\hline $\operatorname{Ag}(\mathrm{I})$ & Triton X-114 & DDTP, $0.1 \mathrm{~mol} \cdot \mathrm{L}^{-1} \mathrm{H}_{2} \mathrm{SO}_{4}$ & 100 \\
\hline $\mathrm{Mn}(\mathrm{II})$ & Triton X-114 & TAN, pH 9.2 & 100 \\
\hline $\mathrm{Pd}(\mathrm{II})$ & Triton X-114 & TAN, pH 8.6 & 99 \\
\hline $\mathrm{Co}(\mathrm{II})$ & Triton X-114 & TAN, pH 3.7 & 98 \\
\hline $\mathrm{Cd}(\mathrm{II})$ & Triton X-114 & DDTP, $0.01 \mathrm{~mol} \cdot \mathrm{L}^{-1} \mathrm{HCl}$ & 99 \\
\hline $\operatorname{Pd}(\mathrm{II})$ & Triton X-114 & DDTP, $0.32 \mathrm{~mol} \cdot \mathrm{L}^{-1} \mathrm{HCl}$ & 101 \\
\hline $\mathrm{Ni}(\mathrm{II})$ & Triton X-114 & TAN, pH 7.0 & 97 \\
\hline $\mathrm{Au}(\mathrm{II})$ & Triton X-114 & DDTP, $1 \mathrm{~mol} \cdot \mathrm{L}^{-1} \mathrm{HCl}$ & 99 \\
\hline $\mathrm{Zn}(\mathrm{II})$ & Triton X-114 & TAN, pH 8.6 & 98 \\
\hline
\end{tabular}

近十几年来, 随着人们对液液相分离研究的不断深 入，离子型表面活性剂的液液相分离体系也被应用于废 水中污染物的提取. 其中, 最常用的是无机酸诱导的阴 离子表面活性剂液液相分离体系. Sicilia 等 ${ }^{[182]}$ 研究了酸 诱导的磺酸盐表面活性剂的液液相分离, 体系形成相分 离所需的 $\mathrm{HCl}$ 浓度随着表面活性剂碳链长度的增加而 降低. 提高盐酸的浓度, 凝聚相对于苯酚的提取效率降
低. 此外, 酸诱导的阴离子表面活性剂液液相分离体系 还可以用于提取水溶液中的芳香化合物 1,4-二氯苯 ${ }^{[142]}$ 、 多环芳烃如范、萠、菲等 ${ }^{[142,183]}$ 、染料分子 brilliant green $^{[184]}$ 以及农药残留分子 ${ }^{[185]}$. 与非离子表面活性剂的 浊点提取法相比，酸诱导的阴离子表面活性剂形成液液 相分离在有机物的提取中，需要的平衡时间短，能够在 较低温度下提取热不稳定的有机物, 表面活性剂分子在 紫外可见光区间没有吸收，对于有机物的定量没有影 响, 缺点是需要的酸浓度较高.

有机盐、无机盐等助剂诱导的离子型表面活性剂形 成的液液相分离体系也被用于提取带电荷的有机 物 ${ }^{[186 ~ 188]}$. Ruiz 等 ${ }^{[186]}$ 研究发现有机盐四丁基溴化铵/烷 基酸表面活性剂在 $\mathrm{p} K_{\mathrm{a}}$ 附近发生液液相分离, 凝聚相能 够有效地富集水溶液中的双酚 $\mathrm{A}$ 、双酚 $\mathrm{F}$ 及相应的甘油 醚. 氢键和疏水相互作用在提取有机物过程中起到重要 的作用. $\mathrm{Na}_{2} \mathrm{SO}_{4}$ /阳离子表面活性剂甲基三辛基氯化铵 Aliquat-336 的液液相分离体系可以有效地富集带负电 的藻毒素 MC-LR 和 MC-YR, 而两性的藻毒素 MC-RR 则不能被提取, 实现了对于带有负电荷的藻毒素分子的 选择性提取 ${ }^{[188]}$.

阴阳离子表面活性剂混合体系形成的液液相分离 也被应用于提取水溶液中挥发性的苯、甲苯、乙苯、卟 啉、甲基橙以及磺胺类分子 ${ }^{[189 \sim 192]}$. Weschayanwiwat 等 ${ }^{[189]}$ 利用阴阳离子表面活性剂 DTAB/十六烷基二苯醚 双磺酸盐(DPDS)混合体系提取苯、甲苯、乙苯等挥发性 有机物, 研究发现, 与非离子表面活性剂相比, DTAB/ DPDS 形成的电中性的囊泡聚集体对于苯有更强的增溶 能力, 驱动力为静电相互作用、疏水相互作用和 $\pi$-阳离 子作用. Chen 等 ${ }^{[192]}$ 研究发现, HFIP 能够诱导 SDS/ DTAB 混合体系在较宽的浓度 $(10 \sim 200 \mathrm{mmol} / \mathrm{L})$ 和比例 (物质的量之比为 $88: 12 \sim 0: 100$ ) 范围内形成液液相分 离, 选取 SDS/DTAB 按物质的量之比 $3: 7$ 混合, 形成的 凝聚相能够通过疏水相互作用、静电相互作用、 $\pi$-阳离 子相互作用以及氢键实现对于磺胺类阴离子化合物的 提取.

复杂液液相分离体系可以用于提取水溶液中的染 料分子. Dubin 课题组 ${ }^{[193]}$ 利用聚二甲基二烯丙基氯化铵 (PDADMAC)/SDS-Triton X-100 液液相分离体系改性后 的多孔玻璃提取水溶液中的 Orange OT, 发现处理过的 多孔玻璃对于 Orange OT 的吸附能力较好, 且在较高的 离子强度下, 吸附率更高. Chiappisi 等 ${ }^{[194,195]}$ 研究发现带 正电荷的壳聚糖 Chitosan 与带有羧酸根的表面活性剂 C18:1E9Ac 在电荷比为 0.2 时, 能够在亚甲基蓝存在的 条件下选择性地提取疏水性染料苏丹红(图 7a), 同时由 于混合体系中羧基的存在, 凝聚相对于金属离子也有很 好的提取作用. 我们课题组 ${ }^{[196]}$ 利用阳离子季铵盐型 Gemini 表面活性剂(12-6-12)与部分水解聚丙烯酰胺 (PAM) 在 $\mathrm{pH} 7.0$ 条件下构筑了液液相分离, 凝聚相能够 
高效、选择性地提取水溶液中的 $\mathrm{MO}$, 且液液相分离体 系改性的石英砂及密胺泡沫对甲基橙具有较好的吸附 效果, 调节溶液 $\mathrm{pH}$ 可以实现染料分子及固定相的循环 利用. 将 Gemini 表面活性剂替换成基于亚胺键的动态 共价表面活性剂, 利用亚胺键的 $\mathrm{pH}$ 响应性, 可以在溶 液中实现凝聚相对于阴离子型染料刚果红(CR)的提取 与释放以及表面活性剂/PAM 的可重复利用(图 7b) ${ }^{[197]}$.

(a)

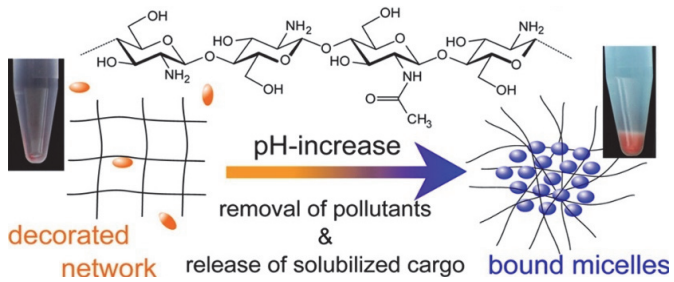

(b)

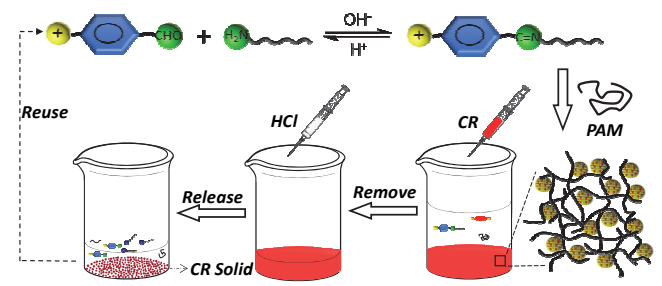

图 7 (a) Chitosan $/ \mathrm{C}_{18: 1} \mathrm{E}_{9} \mathrm{Ac}$ 混合体系提取染料分子的机理 ${ }^{[194,195]}$ 和(b) 动态共价表面活性剂/PAM 形成的凝聚相对于 $\mathrm{CR}$ 的提取和释放过程 的作用机理 ${ }^{[197]}$

Figure 7 (a) Mechanism of extracting dyes by chitosan/ $\mathrm{C}_{18: 1} \mathrm{E}_{9} \mathrm{Ac}$ mixture ${ }^{[194,195]}$ and (b) mechanism of the extraction and release of CR in dynamic covalent surfactant/PAM mixture ${ }^{[197]}$

与浊点提取法相比, 离子型表面活性剂的液液相分 离体系在提取废水中有机物时具有以下优势：离子型表 面活性剂的液液相分离体系能够通过多种作用力实现 极性有机物的富集, 如静电相互作用、疏水相互作用、 氢键、 $\pi$-阳离子相互作用等; 表面活性剂分子组装形成 囊泡、蠕虫状胶束或表面活性剂/聚电解质复合物等聚集 体, 聚集体增大, 分子堆积更加紧密, 能够更好地增溶 有机物; 不需要升温就可以发生相分离, 降低能耗.

\section{5 总结与展望}

表面活性剂的自组装是构建纳米结构最普遍的策 略之一, 在材料科学和分离工程等领域受到关注. 自组 装理论的发展为表面活性剂提取技术提供了良好的理 论基础. 表面活性剂可以通过静电相互作用、疏水相互 作用、氢键、 $\pi$-阳离子相互作用、配位作用等多种方式 实现对于金属离子、疏水性有机物和极性有机物的提取, 极大地扩展了基于表面活性剂提取技术的应用范围. 改 变表面活性剂的结构(如碳链长度、寡聚度等)、浓度以 及改变外界环境(如温度、 $\mathrm{pH}$ 值、无机盐浓度等)能够实 现对于表面活性剂自组装结构和性能的有效调控, 进而 实现对于废水中不同污染物提取效率的调节.

基于表面活性剂的提取技术在废水处理中得到了
长足的发展：胶束增强超滤和固相改性吸附的原理和应 用研究表明，表面活性剂形成的胶束或层状结构有利于 增溶金属离子和有机物，具有高效、低能耗、易于工业 化等特点; 基于液液相分离的提取技术利用表面活性剂 在一定条件下相分离的特点, 实现了对于多种污染物的 富集，在萃取性能和效率上表现出极为明显的优势，在 废水处理和分析领域得到了广泛的关注. 这些提取技术 是基于表面活性剂分子在水溶液中超过临界聚集浓度 能够形成不同聚集体(球形胶束、层状结构)及发生相转 变, 利用聚集体不同区域亲疏水性质的差异, 通过静电 作用、疏水作用、氢键、 $\pi-\pi$ 作用等多重作用力，实现 对于非极性污染物、弱极性污染物以及带电荷污染物的 高效富集. 但是，在实际应用中还有许多问题亟待解决, 例如超滤膜污染、表面活性剂和固定相的回收、对于被 提取物质的选择性、工业化和连续化等问题. 因此，在 今后的研究工作中, 我们应当将新型高效的表面活性剂 用于表面活性剂提取技术，并尽可能提高提取效率和分 离速率. 相信随着研究的不断深入, 基于表面活性剂的 提取方法会不断革新和完善, 进一步推动其在废水处理 中的应用.

\section{作者简介}

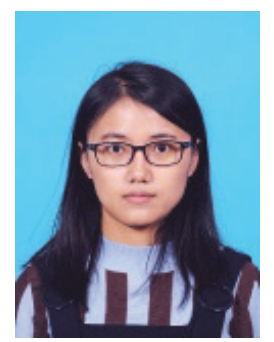

赵微微, 2013 年毕业于山东大学化学与化工学院, 随后加 入中国科学院化学研究所王效琳研究员棵题组, 并于 2018 年 获得物理化学博士学位. 2019 年初至今在清华大学机械工程 系进行博士后研究, 主要从事表面活性剂和聚合物的自组装 及应用研究。

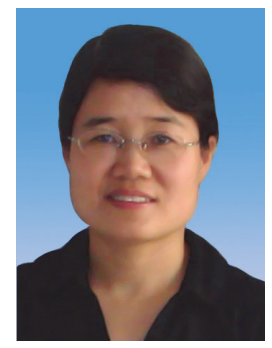

王毅琳, 博士, 中国科学院化学研究所研究员, 中国科学 院大学岗位教授, 博士生导师. 1988 年和 1991 年在兰州大学 化学系分别获得学士和硕士学位; 1997 年在中国科学院化学 研究所获得博士学位; 1997 年 2001 年先后在美国佛罗里达 大学化学系和印第安那一普渡大学化学系从事博士后研究; 2001 年获得中国科学院引进国外杰出人才 “百人计划” 资助; 自 2002 年起在中国科学院化学研究所任研究员. 2010 年获得 
国家杰出青年科学基金资助。研究领域包括新型表面活性剂 的建立与有序组合体的结构和功能调控, 表面活性剂与聚合 物和生物大分子之间的相互作用及其相行为, 表面活性剂在 界面的动态行为等.

\section{References}

[1] Ali, I. Chem. Rev. 2012, 112, 5073.

[2] Clement, R. E.; Yang, P. W. Anal. Chem. 1997, 69, $251 \mathrm{R}$.

[3] Vandevivere, P. C.; Bianchi, R.; Verstraete, W. J. Chem. Technol. Biotechnol. 1998, 72, 289.

[4] Leonard, S. S.; Bower, J. J.; Shi, X. Mol. Cell. Biochem. 2004, 255, 3.

[5] Samanta, S. K.; Singly, O. V.; Jain, R. K. Trends Biotechnol. 2002, $20,243$.

[6] Gupta, V. K.; Carrott, P. J. M.; Ribeiro Carrott, M. M. L.; Suhas. Crit. Rew. Env. Sci. Tec. 2009, 39, 783.

[7] Sethi, S.; Wiesner, M. R. J. Environ. Eng. Div. ASCE 1995, 121, 874.

[8] Eliassen, R.; Bennet, G. E. J. Water Pollut. Control Fed. 1967, 39, R82.

[9] Lee, D. W.; Hong, W. H.; Hwang, K. Y. Sep. Sci. Technol. 2000, 35, 1951.

[10] Hempfling, C. Environ. Prog. 1997, 16, 164.

[11] Fox, M. A.; Dulay, M. T. Chem. Rev. 1993, 93, 341.

[12] Nicolet, L.; Rott, V. Water Sci. Technol. 1999, 40, 191.

[13] LaPara, T. M.; Konopka, A.; Nakatsu, C. H.; Alleman, J. E. J. Environ. Eng. ASCE 2000, 126, 739.

[14] Yang, X.; Cai, H.; Bao, M.; Yu, J.; Lu, J.; Li, Y. Chin. J. Chem. 2017, 35, 1549.

[15] Huang, X.; Wang, W.; Ling, L.; Zhang, W. Acta Chim. Sinica 2017, 75, 529. (黄潇月，王伟，凌岗，张伟贤，化学学报, 2017, 75, 529.)

[16] Zhou, L. Acta Chim. Sinica 2017, 75, 552. (周立祥, 化学学报, 2017, 75, 552.)

[17] Tang, J.; Tang, L.; Feng, H.; Dong, H.; Zhang, Y.; Liu, S.; Zeng, G. Acta Chim. Sinica 2017, 75, 575. (汤晶, 汤琳, 冯浩朋, 董浩然, 章毅，刘思诗，曾光明，化学学报, 2017, 75, 575.)

[18] Xia, X.; Hua, Y.; Huang, X.; Ling, L.; Zhang, W. Acta Chim. Sinica 2017, 75, 594. (夏雪芬, 滑熠龙, 黄㴋月, 凌岗, 张伟贤, 化学学 报, 2017, 75, 594.)

[19] Jia, F.; Liu, J.; Zhang, L. Acta Chim. Sinica 2017, 75, 602. (贾法龙, 刘娟, 张礼知, 化学学报, 2017, 75, 602.)

[20] Zhao, G.; Zhu, B. Principles of Surfactant Action, Chinese Light Industry Press, Beijing, 2003. (赵国胥, 朱步瑶, 表面活性剂作用 原理, 中国轻工业出版社, 北京, 2003.)

[21] Fan, Y.; Han, Y.; Wang, Y. L. Acta Phys.-Chim. Sin. 2016, 32, 214. (范雅珣, 韩玉淳, 王毅琳, 物理化学学报, 2016, 32, 214.)

[22] Gibbs, B. F.; Kermasha, S.; Alli, I.; Mulligan, C. N. Int. J. Food Sci. Nutr. 1999, 50, 213.

[23] Hinze, W. L.; Pramauro, E. Crit. Rev. Anal. Chem. 1993, 24, 133.

[24] Israelachvili, J. N.; Mitchell, D. J.; Ninham, B. W. J. Chem. Soc., Faraday Trans. 2 1976, 72, 1525.

[25] Ravi Kumar, M. N. V. React. Funct. Polym. 2000, 46, 1.

[26] Rosen, M. J. Surfactants and Interfacial Properties, John Wiley\&Sons, New York, 1978.

[27] Tanford, C. The Hydrophobic Effect: Formation of Micelles and Biological Membranes, Wiley, New York, 1980.

[28] Zana, R. Structure-Performance Relationships in Surfactants, Marcel Dekker, New York, 1997.

[29] Pastrana-Martínez, L. M.; Morales-Torres, S.; Figueiredo, J. L.; Faria, J. L.; Silva, A. M. T. Water Res. 2015, 77, 179.

[30] Syafei, A. D.; Lin, C.-F.; Wu, C.-H. J. Colloid Interf. Sci. 2008, 323, 112.

[31] Secondes, M. F. N.; Naddeo, V.; Belgiorno, V.; Ballesteros-Jr, F. J. Harzard. Mater. 2014, 264, 342.

[32] Ren, Y.; Lia, T.; Zhang, W.; Wang, S.; Shi, M.; Shan, C.; Zhang, W.; Guan, X.; Lv, L.; Hua, M.; Pan, B. J. Harzard. Mater. 2019, 365 , 312.

[33] Choi, Y.-K.; Lee, S.-B.; Lee, D.-J.; Ishigami, Y.; Kajiuchi, T. J. Membr. Sci. 1998, 148, 185.

[34] Xu, K.; Zeng, G.-M.; Huang, J.-H.; Wu, J.-Y.; Fang, Y.-Y.; Huang, G.; Li, J.; Xi, B.; Liu, H. Colloids Surf., A 2007, 294, 140

[35] Gzara, L.; Dhahbi, M. Desalination 2001, 137, 241.
[36] Ahmad, A. L.; Puasa, S. W. Chem. Eng. J. 2007, 132, 257.

[37] Bielska, M.; Szymanowski, J. Water Res. 2006, 40, 1027.

[38] Talens-Alesson, F. I.; Urbaski, R.; Szymanowski, J. Colloids Surf., A 2001, 178, 71.

[39] Xiarchos, I.; Jaworska, A.; Zakrzewska-Trznadel, G. J. Membr. Sci. 2008, 321, 222.

[40] Landaburu-Aguirre, J.; Pongracz, E.; Peramaki, P.; Keiski, R. L. J. Hazard. Mater. 2010, 180, 524.

[41] Landaburu-Aguirre, J.; García, V.; Pongrácz, E.; Keiski, R. L. Desalination 2009, 240, 262.

[42] Son, G.; Lee, S. Korean J. Chem. Eng. 2011, 28, 793

[43] Rafique, R. F.; Chowdhury, Z. Z.; Moon, J.; Lee, S. Int. J. Innov. Eng. Technol. 2018, 10, 112.

[44] Channarong, B.; Lee, S. H.; Bade, R.; Shipin, O. V. Desalination 2010, 262, 221

[45] Scamehorn, J. F.; Christian, S. D.; El-Sayed, D. A.; Uchiyama, H.; Younis, S. S. Sep. Sci. Technol. 1994, 29, 809.

[46] Li, X.; He, S.; Feng, C.; Zhu, Y.; Pang, Y.; Hou, J.; Luo, K.; Liao, X. Sustainability 2018, 10, 92.

[47] Kim, H.; Baek, K.; Kim, B.-K.; Shin, H.-J.; Yang, J.-W. Korean J. Chem. Eng. 2008, 25, 253.

[48] Schwarze, M.; Groß, M.; Moritz, M.; Buchner, G.; Kapitzki, L.; Chiappisi, L.; Gradzielski, M. J. Membr. Sci. 2015, 478, 140

[49] Yang, H. S.; Han, K. H.; Kang, D. W.; Kim, Y. H. Korean J. Chem. Eng. 1996, 13, 448.

[50] Akita, S.; Castillo, L. P.; Nii, S.; Takahashi, K.; Takeuchi, H. J. Membr. Sci. 1999, 162, 111.

[51] Ferella, F.; Prisciandaro, M.; De Michelis, I.; Veglio, F. Desalination 2007, 207, 125.

[52] Huang, J.; Li, H.; Zeng, G.; Shi, L.; Gu, Y.; Shi, Y.; Tang, B.; Li, X. Sep. Purif. Technol. 2018, 207, 199.

[53] Nekoo, A. B.; Khamforoush, M. Iran. Polym. J. 2019, 28, 391.

[54] Bade, R; Lee, S. H.; Jo, S.; Lee, H.-S.; Lee, S.-E. Desalination 2008, 229,264

[55] Baek, K.; Yang, J.-W. J. Harzard. Mater. 2004, 108, 119.

[56] Abbasi-Garravand, E.; Mulligan, C. N. Sep. Purif. Technol. 2014, 132, 505.

[57] Bahmani, P.; Maleki, A.; Rezaee, R.; Mahvi, A. H.; Khamforoush, M.; Athar, S. D.; Daraei, H.; Gharibi, F.; McKay, G. J. Environ. Health. Sci. Eng. 2019, 17, 115.

[58] Baek, K.; Kim, B.-K.; Cho, H.-J.; Yang, J.-W. J. Harzard. Mater 2003, 99, 303.

[59] Zaghbani, N.; Hafiane, A.; Dhahbi, M. Desalination 2008, 222, 348.

[60] Bielska, M.; Prochaska, K. Dyes Pigm. 2007, 74, 410.

[61] Huang, J.-H.; Zhou, C.-F.; Zeng, G.-M.; Li, X.; Niu, J.; Huang, H.-J.; Shi, L.-J.; He, S.-B. J. Membr. Sci. 2010, 365, 138.

[62] Wasan, D. T.; Ginn, M. E.; Shah, D. O. Surfactant Science Series: Surfactants in Chemical/Process, Marcel Dekker, New York, 1998.

[63] Purkait, M. K.; DasGupta, S.; De, S. Sep. Purif. Technol. 2004, 37, 81.

[64] Purkait, M. K.; DasGupta, S.; De, S. J. Colloid Interface Sci. 2004, $270,496$.

[65] Schwarze, M.; Schaefer, L.; Chiappisi, L.; Gradzielski, M. Sep. Purif. Technol. 2018, 199, 20.

[66] Rubio, S.; Pérez-Bendito, D. TrAC, Trends Anal. Chem. 2003, 22, 470.

[67] Yin, C. Y.; Aroua, M. K.; Ashri, W. M.; Daud, W. Sep. Purif. Technol. 2007, 52, 403

[68] Bhatnagar, A.; Hogland, W.; Marques, M.; Sillanpää, M. Chem. Eng. J. 2013, 219, 499.

[69] Reeve, P. J.; Fallowfield, H. J. J. Environ. Manage. 2018, 205, 253

[70] Jiménez-Castañeda, M. E.; Medina, D. I. Water 2017, 9, 235.

[71] Zhu, R.; Chen, Q.; Zhou, Q.; Xi, Y.; Zhu, J.; He, H. Appl. Clay Sci. 2016, 123, 239 .

[72] Ahn, C. K.; Park, D.; Woo, S. H.; Park, J. M. J. Hazard. Mater 2009, 164, 1130 .

[73] Farooq, W.; Hong, H.-J.; Kim, E. J.; Yang, J.-W. Sep. Sci. Technol. 2012, 47, 1906.

[74] Chen, W.-F.; Zhang, Z.-Y.; Li, Q.; Wang, H.-Y. Chem. Eng. J. 2012 203, 319.

[75] Hong, H.-J.; Kim, H.; Baek, K.; Yang, J.-W. Desalination 2008, 223 221.

[76] Monser, L.; Adhoum, N. Sep. Purif. Technol. 2002, 26, 137.

[77] Mohamed, M. M. J. Colloid Interface Sci. 2004, 272, 28

[78] Choi, H. D.; Shin, M. C.; Kim, D. H.; Jeon, C. S.; Baek, K. 
Desalination 2008, 223, 290.

[79] Mahmoud, M. E.; Nabil, G. M.; El-Mallah, N. M.; Karar, S. B. Desalin. Water Treat. 2016, 57, 8389.

[80] Nabil, G. M.; El-Mallah, N. M.; Mahmoud, M. E. J. Ind. Eng. Chem. 2014, 20, 994.

[81] Dong, Y.; Wu, D.; Chen, X.; Lin, Y. J. Colloid Interface Sci. 2010, 348,585 .

[82] Ghiaci, M.; Kia, R.; Abbaspur, A.; Seyedeyn-Azad, F. Sep. Purif. Technol. 2004, 40, 285.

[83] Bowman, R. S. Microporous Mesoporous Mater. 2003, 61, 43.

[84] Macedo-Miranda, M. G.; Olguín, M. T. J. Inclusion Phenom. Macrocyclic Chem. 2007, 59, 131.

[85] Chutia, P.; Kato, S.; Kojima, T.; Satokawa, S. J. Hazard. Mater. 2009, 162, 204.

[86] Widiastuti, N.; Wu, H.; Ang, M.; Zhang, D.-K. Desalination 2008, $218,271$.

[87] Naghash, A.; Nezamzadeh-Ejhieh, A. J. Ind. Eng. Chem. 2015, 31, 185.

[88] Wingenfelder, U.; Furrer, G.; Schulin, R. Microporous Mesoporous Mater. 2006, 95, 265

[89] Haggerty, G. M.; Bowman, R. S. Environ. Sci. Technol. 1994, 28, 452.

[90] Liu, J.; Huang, H.; Huang, R.; Zhang, J. Z.; Hao, S. S.; Shen, Y. Y.; Chen, H. Water Environ. Res. 2016, 88, 490.

[91] Cortés-Martínez, R.; Martínez-Miranda, V.; Solache-Ríos, M.; García-Sosa, I. Sep. Sci. Technol. 2004, 39, 2711.

[92] Torabian, A.; Kazemian, H.; Seifi, L.; Bidhendi, G. N.; Azimi, A. A.; Ghadiri, S. K. Clean: -Soil, Air, Water 2010, 38, 77.

[93] Abatal, M.; Olguin, M. T. Environm. Earth Sci. 2013, 69, 2691.

[94] Elsheikh, A. F.; Ahmad, U. K.; Ramli, Z. Desalin. Water Treat. 2016, 57, 8302.

[95] Benkli, Y. E.; Can, M. F.; Turan, M.; Çelik, M. S. Water Res. 2005, $39,487$.

[96] Tomašević-Čanović, M.; Daković, A.; Rottinghaus, G.; Matijašević, S.; Đuričić, M. Microporous Mesoporous Mater. 2003, 61, 173.

[97] Nikashina, V. A.; Myasoedov, B. F. In Natural Microporous Materials in Environmental Technology, Eds.: Misaelides, P.; Macášek, F.; Pinnavaia, T. J.; Colella, C., Springer Netherlands, Dordrecht, 1999, p. 335.

[98] Xie, Q.; Lin, Y.; Wu, D.; Kong, H. Fuel 2017, 203. 411.

[99] Shirzadi, H.; Nezamzadeh-Ejhieh, A. J. Mol. Liq. 2017, 230, 221.

[100] Trana, H. N.; Vietb, P. V.; Chao, H.-P. Ecotoxicol. Environ. Saf. 2018, $147,55$.

[101] Liu, C. M.; Wu, P. X.; Zhu, Y. J.; Tran, L. T. Chemosphere 2016, 144,1026

[102] Jordan, J. W. J. Phys. Chem. 1949, 53, 294.

[103] Smith, J. A.; Galan, A. Environ. Sci. Technol. 1995, 29, 685.

[104] Shen, Y. Chemosphere 2001, 44, 989.

[105] Zhu, L.; Chen, B. Environ. Sci. Technol. 2000, 34, 2997.

[106] Zhu, L.; Chen, B.; Shen, X. Environ. Sci. Technol. 2000, 34, 468.

[107] Zhu, L.; Li, Y.; Zhang, J. Environ. Sci. Technol. 1997, 31, 1407.

[108] Ma, J.; Zhu, L. Chemosphere 2007, 68, 1883.

[109] Wu, Z.; Zhu, L. J. Environ. Sci.-China 2012, 24, 248.

[110] Nourmoradi, H.; Khiadani, M.; Nikaeen, M. J. Chem. 2013, 2013, 1.

[111] Chen, D. M.; Chen, J.; Luan, X. L.; Ji, H. P.; Xia, Z. G. Chem. Eng. $J$. 2011, 171, 1150 .

[112] Chen, D. M.; Chen, J.; Wang, X. M.; Luan, X. L.; Ji, H. P.; Xu, F. Adv. Mater. Res. 2011, 178, 29.

[113] Wang, L.; Wang, A. J. Hazard. Mater. 2008, 160, 173.

[114] Acisli, O.; Khataee, A.; Karaca, S.; Sheydaei, M. Ultrason. Sonochem. 2016, 31, 116.

[115] Lin, S.-H.; Juang, R.-S. J. Hazard. Mater. 2002, 92, 315.

[116] Malakul, P.; Srinivasan, K. R.; Wang, H. Y. Ind. Eng. Chem. Res. 1998, 37, 4296.

[117] Li, S.-Z.; Wu, P.-X. J. Hazard. Mater. 2010, 173, 62.

[118] Özcan, A. S.; Gök, Ö.; Özcan, A. J. Hazard. Mater. 2009, 161, 499.

[119] Zhu, R.; Zhu, L.; Zhu, J. Environ. Sci. 2006, 27, 91. (朱润良, 朱利 中, 朱建喜, 环境科学, 2006, 27, 91.)

[120] Ma, J.; Zhu, L. J. Harzard. Mater. 2006, 136, 982.

[121] Bungenberg de Jong, H. G. Colloid Science, Elsevier, Amsterdam, 1949.

[122] Bohidar, H. B. J. Surface Sci. Technol. 2008, 24, 105.

[123] Veis, A. Adv. Colloid Interface Sci. 2011, 167, 2.

[124] Kizilay, E.; Kayitmazer, A. B.; Dubin, P. L. Adv. Colloid Interface Sci. 2011, 167, 24

[125] Michaeli, I.; Overbeek, J. T. G.; Voorn, M. J. J. Polym. Sci. 1957, 23,
443.

[126] Sato, H.; Nakajima, A. Colloid. Polym. Sci. 1974, 252, 944.

[127] Wang, M.; Wang, Y. L. Soft Matter 2014, 10, 7909.

[128] Zhao, W.; Wang, Y. L. Adv. Colloid Interface Sci. 2017, 239, 199.

[129] Ruan, K.; Xiao, J.; Zhang, L.; Zhao, Z.; Zhang, Y. Acta Chim. Sinica 2002, 60, 961. (阮科, 肖进新, 张翎, 赵振国, 张禹负, 化学学报, 2002, 60,961.)

[130] Corti, M.; Degiorgio, V. Phys. Rev. Lett. 1985, 55, 2005.

[131] Lindman, B.; Carlsson, A.; Karlström, G.; Malmsten, M. Adv. Colloid Interface Sci. 1990, 32, 183.

[132] Cohen, I.; Economou, P. J. Phys. Chem. 1964, 68, 2801.

[133] Cohen, I.; Economou, P. J. Am. Oil Chem. Soc. 1964, 41, 461.

[134] Cohen, I.; Economou, P.; Libackyj, A. J. Phys. Chem. 1962, 66, 1829.

[135] Cohen, I.; Hiskey, C. F.; Oster, G. J. Colloid Sci. 1954, 9, 243.

[136] Cohen, I.; Vassiliades, T. J. Phys. Chem. 1961, 65, 1774.

[137] Cohen, I.; Vassiliades, T. J. Am. Oil Chem. Soc. 1962, 39, 246.

[138] Wang, M.; Fan, Y.; Han, Y.; Nie, Z.; Wang, Y. L. Langmuir 2013, 29, 14839.

[139] Hoffmann, H.; Thunig, C.; Munkert, U.; Meyer, H. W.; Richter, W. Langmuir 1992, 8, 2629.

[140] Horbaschek, K.; Hoffmann, H.; Thunig, C. J. Colloid Interface Sci. 1998, 206, 439 .

[141] Casero, I.; Sicilia, D.; Rubio, S.; Pérez-Bendito, D. Anal. Chem. 1999, 71, 4519.

[142] Paleologos, E. K.; Giannakopoulos, S. S.; Zygoura, P. D.; Kontominas, M. G. J. Agric. Food Chem. 2006, 54, 5236.

[143] Li, Y.; Dubin, P. L.; Havel, H. A.; Edwards, S. L.; Dautzenberg, H. Langmuir 1995, 11, 2486.

[144] Wang, Y. L.; Kimura, K.; Dubin, P. L.; Jaeger, W. Macromolecules 2000, 33, 3324

[145] Wang, Y. L.; Kimura, K.; Huang, Q.; Dubin, P. L.; Jaeger, W. Macromolecules 1999, 32, 7128.

[146] Dubin, P. L.; Li, Y.; Jaeger, W. Langmuir 2008, 24, 4544.

[147] Löf, D.; Niemiec, A.; Schillén, K.; Loh, W.; Olofsson, G. J. Phys. Chem. B 2007, 111, 5911.

[148] Carlsson, A.; Karlströem, G.; Lindman, B. Langmuir 1986, 2, 536.

[149] Hashidzume, A.; Ohara, T.; Morishima, Y. Langmuir 2002, 18, 9211.

[150] Thalberg, K.; Lindman, B.; Bergfeldt, K. Langmuir 1991, 7, 2893.

[151] Thalberg, K.; Lindman, B.; Karlströem, G. J. Phys. Chem. 1991, 95 , 3370 .

[152] Watanabe, H.; Tanaka, H. Talanta 1978, 25, 585.

[153] Bordier, C. J. Biol. Chem. 1981, 256, 1604.

[154] Ballesteros-Gómez, A.; Sicilia, M. D.; Rubio, S. Anal. Chim. Acta 2010, 677, 108 .

[155] Trakultamupatam, P.; Scamehorn, J. F.; Osuwan, S. Sep. Sci. Technol. 2002, 37, 1291.

[156] Kungsanant, S.; Kittisrisawai, S.; Suriya-Amrit, P.; Kitiyanan, B.; Chavadej, S.; Osuwan, S.; Scamehorn, J. F. Sep. Sci. Technol. 2018, 53, 2662.

[157] Pino, V.; Ayala, J. H.; Afonso, A. M.; González, V. J. Chromatogr. A 2002, 949, 291.

[158] Song, G. Q.; Lu, C.; Hayakawa, K.; Lin, J.-M. Anal. Bioanal. Chem. 2006, 384, 1007.

[159] Taechangam, P.; Scamehorn, J. F.; Osuwan, S.; Rirksomboon, T. Colloids Surf., A 2009, 347, 200.

[160] Mahugo Santana, C.; Sosa Ferrera, Z.; Santana Rodriguez, J. J. Analyst 2002, 127, 1031.

[161] Haddou, B.; Canselier, J. P.; Gourdon, C. Sep. Purif. Technol. 2006, $50,114$.

[162] Ghouas, H.; Haddou, B.; Kameche, M.; Canselier, J. P.; Gourdon, C. J. Surfactants Deterg. 2016, 19, 57.

[163] Pourreza, N.; Rastegarzadeh, S.; Larki, A. Talanta 2008, 77, 733.

[164] Liu, W.; Zhao, W.; Chen, J.; Yang, M. Anal. Chim. Acta 2007, 605, 41.

[165] Appusamy, A.; John, I.; Ponnusamy, K.; Ramalingam, A. Eng. Sci. Technol. Int. J. 2014, 17, 137.

[166] Zhou, J.; Chen, J.; Cheng, Y.; Li, D.; Hu, F.; Li, H. Talanta 2009, 79, 189.

[167] Lee, C.-K.; Su, W.-D. Sep. Sci. Technol. 1999, 34, 3267.

[168] Andreia Mesquita da Silva, M.; Lúcia Azzolin Frescura, V.; José Curtius, A. Spectrochim. Acta, Part B 2001, 56, 1941.

[169] Chen, J.; Teo, K. C. Anal. Chim. Acta 2001, 450, 215.

[170] Chen, J.; Teo, K. C. Anal. Chim. Acta 2001, 434, 325.

[171] Lázaro Gallindo Borges, D.; Mesquita Silva da Veiga, M. A.; Azzolin Frescura, V. L.; Welz, B.; Curtius, A. J. J. Anal. At. 
Spectrom. 2003, 18, 501.

[172] Manzoori, J. L.; Bavili-Tabrizi, A. Anal. Chim. Acta 2002, 470, 215.

[173] Manzoori, J. L.; Karim-Nezhad, G. Anal. Chim. Acta 2003, 484, 155

[174] Manzoori, J. L.; Karim-Nezhad, G. Anal. Sci. 2003, 19, 579.

[175] Teo, K. C.; Chen, J. Analyst 2001, 126, 534.

[176] Satti, A. A.; Durukan Temuge, I.; Bektas, S.; Arpa Şahin, Ç. Turk. J. Chem. 2016, 40, 979

[177] Trakultamupatam, P.; Scamehorn, J. F.; Osuwan, S. Sep. Sci. Technol. 2002, 37, 1291.

[178] Pourreza, N.; Zareian, M. J. Hazard. Mater. 2009, 165, 1124.

[179] de Wuilloud, J. C. A.; Wuilloud, R. G.; Sadi, B. B. M.; Caruso, J. A. Analyst 2003, 128, 453 .

[180] Kukusamude, C.; Santalad, A.; Boonchiangma, S.; Burakham, R.; Srijaranai, S.; Chailapakul, O. Talanta 2010, 81, 486.

[181] Zarei, A. R. Anal. Biochem. 2007, 369, 161.

[182] Sicilia, D.; Rubio, S.; Pérez-Bendito, D. Anal. Chim. Acta 2002, $460,13$.

[183] Goryacheva, I. Y.; Shtykov, S. N.; Loginov, A. S.; Panteleeva, I. V. Anal. Bioanal. Chem. 2005, 382, 1413.

[184] Hosseini, S. S. S.; Khezri, S.; Khosravi, A. Appl. Water Sci. 2018, 8, 109.

[185] Jia, G.; Bi, C.; Wang, Q.; Qiu, J.; Zhou, W.; Zhou, Z. Anal. Bioanal.
Chem. 2006, 384, 1423

[186] Ruiz, F. J.; Rubio, S.; Perez-Bendito, D. J. Chromatogr. A 2007, $1163,269$.

[187] Moral, A.; Sicilia, M. D.; Rubio, S. J. Chromatogr. A 2009, 1216 , 3740.

[188] Man, B. K. W.; Lam, M. H. W.; Lam, P. K. S.; Wu, R. S. S.; Shaw, G. Environ. Sci. Technol. 2002, 36, 3985.

[189] Weschayanwiwat, P.; Krutlert, D.; Scamehorn, J. F. Sep. Sci. Technol. 2009, 44, 2582.

[190] Weschayanwiwat, P.; Kunanupap, O.; Scamehorn, J. F. Chemosphere 2008, 72, 1043.

[191] Akama, Y.; Tong, A.-J.; Ito, M.; Tanaka, S. Talanta 1999, 48, 1133.

[192] Chen, D.; Zhang, P.; Li, Y.; Mei, Z.; Xiao, Y. Anal. Bioanal. Chem. 2014, 406, 6051 .

[193] Wang, Y. L.; Banziger, J.; Dubin, P. L.; Filippelli, G.; Nuraje, N. Environ. Sci. Technol. 2001, 35, 2608.

[194] Chiappisi, L.; Prévost, S.; Grillo, I.; Gradzielski, M. Langmuir 2014, 30,1778

[195] Chiappisi, L.; Simon, M.; Gradzielski, M. ACS Appl. Mater. Interfaces 2015, 7, 6139.

[196] Zhao, W.; Fan, Y.; Wang, H.; Wang, Y. L. Langmuir 2017, 33, 6846.

[197] Zhao, W.; Wang, H.; Wang, Y. L. Soft Matter. 2018, 14, 4178.

(Zhao, C.) 